\title{
COVID-19. Complicaciones del control prenatal y resultado perinatal
}

\author{
Juan Caripidis, ${ }^{1}$ (D) Evelyn Álvarez, ${ }^{2}$ (D) Maryori Zapata. ${ }^{2}$
}

\begin{abstract}
RESUMEN
Existen pocos datos sobre el impacto del SARS-CoV-2 en el embarazo. Se presentan cuatro casos de infección por SARS-CoV-2, confirmados por reacción en cadena de la polimerasa con transcripción inversa en tiempo real de secreciones nasofaringeas recolectadas por hisopos, en embarazadas con síntomas respiratorios, que acudieron a la consulta de control prenatal y perinatología del autor, en el Hospital de Clínicas Caracas. La evolución clínica fue aguda para SARSCoV-2. Permanecieron hospitalizadas siete dias, en dos de ellas se mantuvo vigilancia maternofetal estricta, comprobándose el bienestar fetal. La resolución obstétrica fue vía cesárea, a las 40 semanas, con evolución satisfactoria. A uno de los recién nacidos se le tomó muestra de cordón umbilical para serología de SARS-CoV-2 al nacimiento, resultando la inmunoglobulina $G$ positiva y la $M$ negativa. El análisis de estos datos es importante para orientar la práctica clínica, abarcando la prevención, detección, aislamiento, investigación epidemiológica, diagnóstico y tratamiento.
\end{abstract}

Palabras clave: SARS-CoV-2, COVID-19, control prenatal, resultado perinatal

\section{SUMMARY}

There is little data on the impact of SARS-CoV-2 on pregnancy. There are four cases of SARS-CoV-2 infection, confirmed by polymerase chain reaction with real-time reverse transcription of nasopharyngeal secretions collected by swabs, in pregnant women with respiratory symptoms, who attended the author's prenatal care and perinatology consultation at the Hospital de Clinicas Caracas. The clinical evolution was acute for SARSCoV-2. They remained hospitalized for seven days, in two of them strict maternal and fetal surveillance was maintained, checking fetal wellbeing. Obstetric resolution was via cesarean section at 40 weeks, with satisfactory evolution. One of the newborns was sampled for SARS-CoV-2 serology for umbilical cord at birth, resulting in positive immunoglobulin $G$ and $M$ negative. The analysis of these data is important to guide clinical practice, covering prevention, detection, isolation, epidemiological investigation, diagnosis and treatment.

Keywords: SARS-CoV-2, COVID-19, prenatal care, perinatal outcome.

\section{INTRODUCCIÓN}

Los coronavirus son históricamente conocidos por causar en forma endémica cuadros de resfrío común, sin embargo, en las últimas décadas han emergido dos betacoronavirus de importancia epidemiológica, causantes del síndrome respiratorio agudo severo (SARS-CoV- 1) y el síndrome respiratorio del medio

\footnotetext{
${ }^{1}$ Especialista en Obstetricia y Ginecología / Perinatología. Hospital de Clínicas Caracas. ${ }^{2}$ Especialistas en Obstetricia y Ginecología.
}

oriente (MERS-CoV), los cuales pueden causar neumonía grave, insuficiencia respiratoria y muerte (1).

El grupo 2 del género betacoronavirus (CoV-2) incluye varios subgrupos, destacando OC43 y HKU1, el SARS-CoV-1, el MERS-CoV y, el más recientemente identificado, el coronavirus 2, del síndrome respiratorio agudo severo (SARS-CoV-2), que es el coronavirus causante de la enfermedad llamada COVID-19 (2-4).

La COVID-19, causada por el SARS-CoV-2 es una enfermedad altamente infecciosa que fue descrita por 
primera vez por Huang y cols. (5), cuyo informe se centró principalmente en las principales características y resultados de las adultas no embarazadas. El primer estudio de cohorte publicado sobre el embarazo describió a 55 mujeres embarazadas infectadas con COVID-19, de las cuales solo una tuvo una presentación grave y no hubo muertes (6). Aún se desconoce mucho sobre el impacto de la enfermedad en el embarazo, las diferencias en el curso clínico y los resultados en esta población, así como el riesgo de transmisión vertical.

Desde el punto de vista clínico la gestante suele tener infecciones respiratorias de mayor gravedad, en contraposición a las no gestantes, relacionadas con evolución adversa del embarazo y alta morbimortalidad maternofetal por los cambios fisiológicos del embarazo, tales como actividad reducida de las células natural killer, los macrófagos inflamatorios y las células $T$, con factores mecánicos y bioquímicos que afectan el intercambio gaseoso y la función pulmonar, con capacidad residual funcional y volumen residual disminuidos, especialmente en el tercer trimestre (68).

En el SARS y el MERS, hasta el $35 \%$ y el $41 \%$ de las pacientes embarazadas requirieron ventilación mecánica y las tasas de mortalidad alcanzaron el $18 \%$ y el $25 \%$, respectivamente $(6,9,10)$. Por el contrario, la mayoría de los casos notificados de infección por COVID-19 en pacientes embarazadas han mostrado un curso leve o asintomático de la enfermedad (10).

Hasta la fecha, no hay evidencia concreta de si existe transmisión intrauterina del SARS-CoV-2. Se han reportado casos de neonatos con pruebas positivas de reacción en cadena de la polimerasa en tiempo real (RT-PCR) poco después del parto, con un riesgo global estimado de transmisión vertical de $1 \%(5-8,11,12)$.
El primer caso diagnosticado de forma oficial en Latinoamérica ocurrió en Ecuador, en febrero de 2020. A partir de ese momento, se han descrito casos de forma progresiva en todos los países latinoamericanos, con un factor epidemiológico común en personas provenientes de España, Italia y China (1).

La evidencia de los casos reportados a nivel mundial es controversial, con relación a la mayor susceptibilidad de las gestantes a la infección por SARS CoV-2 y al aumento de riesgo en desarrollar neumonía con relación al resto de la población. Además, hay preocupación por los efectos adversos en los resultados perinatales y la programación fetal (13), en particular ante la emergencia de nuevas variantes del SARS-CoV-2 en los diversos países afectados, con protocolos cambiantes de forma dinámica en medidas de bioseguridad, profilaxis, diagnóstico y terapéutica (14-22). Los estudios son todavía limitados en lo que respecta a la gestación y la COVID-19, por lo que se necesita registrar y reportar cuando se presenten casos con complicaciones maternas y perinatales asociadas a COVID-19 (14-22).

Si bien existen pautas nacionales (21) e internacionales $(23,24)$ por el Ministerio del Poder Popular para la Salud (MPPS) y la Organización Mundial de la Salud (OMS), sobre la definición de caso confirmado utilizando pruebas diagnósticas como la reacción en cadena de la polimerasa (PCR) o pruebas de antígenos, dichos documentos técnicos enfatizan la necesidad de considerar aspectos clínicos, marcadores bioquímicos, hallazgos radiológicos y antecedentes epidemiológicos de manera conjunta, para no diferir el inicio del tratamiento de casos sospechosos o probables. De igual forma, la "Red COVID-19 y Gestación" (25), como grupo multidisciplinario que discute la evidencia relacionada con la pandemia por COVID-19, además de la promoción de la investigación y facilitación de acceso a información científica en la gestación en medio de la pandemia ha concluido en medio del 
debate de su plataforma digital que para la definición de diagnóstico de COVID-19 tanto en paciente gestante como no gestante, no es criterio absoluto la presencia de una prueba positiva por PCR o pruebas de antígenos, dada una considerable tasa de falsos negativos, que varía según el día de la infección clínica o subclínica por SARS-CoV-2, entre otros factores intervinientes, como aquellos relacionados con la toma, transporte y procesamiento de la muestra, así como la calidad del laboratorio responsable de realizar la prueba. En algunos pacientes, el ARN viral solo puede detectarse durante algunos días, mientras que en otros se puede detectar durante varias semanas, incluso meses (24).

El siguiente reporte de serie de casos de gestantes con COVID-19 se realizó con la finalidad de analizar las complicaciones maternas y el resultado perinatal observado en la consulta de control prenatal particular de los autores, en el Hospital de Clínicas Caracas.

\section{REPORTE DE CASOS}

Entre los meses de mayo de 2020 y mayo de 2021, se evaluaron 21 pacientes con diagnóstico de COVID-19, con hallazgos clínicos, radiológicos, bioquímicos, y epidemiológicos característicos de la enfermedad y/o confirmación por PCR o pruebas de antígenos. Entre ellas, se les realizó seguimiento del embarazo según las recomendaciones del consenso de la Sociedad Venezolana de Ultrasonido en Medicina (AVUM) para gestantes con COVID-19 (20), en 17 casos, en vista de afectación asintomática-leve, con evolución materna y fetal satisfactoria, sin eventualidades periparto, con recién nacidos a término con peso al nacer adecuado para edad gestacional.

Cuatro pacientes diagnosticadas en el tercer trimestre, ameritaron hospitalización por afectación moderada-severa materna, sin requerir ingreso a la unidad de cuidados intensivos (UCI), recibieron tratamiento antiviral con remdesivir, oxigenoterapia, anticoagulación y tratamiento con corticoesteroides, con posterior alta por mejoría materna y ausencia de compromiso materno, con un tiempo de hospitalización de 7-10 días. Uno de los cuatro casos actualmente sigue con el curso de su gestación, con resolución obstétrica pendiente al término, sin secuelas maternas y sin evidencia de alteración fetal en los estudios de seguimiento. En cuanto a los otros 3 casos, en 2 de ellos se presentó resolución obstétrica espontánea al término por parto sin alteraciones.

Con respecto al caso restante, se trataba de paciente femenino de 37 años de edad, I gesta, con fecha de última regla incierta, con embarazo de 33 semanas más 1 día, quien consultó por emergencia el día $25 / 01 / 2021$ por presentar fiebre, cuantificada en $39^{\circ}$, que atenuaba con la administración de acetaminofén, concomitantemente presentó intolerancia a la vía oral y dificultad para respirar, motivo por el cual se evaluó y se decidió su egreso con tratamiento ambulatorio sintomático con antipiréticos y antieméticos, en vista de la ausencia de hallazgos positivos al examen físico. El día 29/01/2021 acudió nuevamente por persistir la sintomatología y asociarse disnea a leves esfuerzos $\mathrm{y}$ tos seca. Consignó PCR para SARS-CoV-2 negativa, de fecha 22/01/2021. Al examen físico de ingreso, presentaba tensión arterial (TA) 103/67 $\mathrm{mmHg}$, frecuencia cardiaca (FC) 99 1pm, frecuencia respiratoria (FR) $20 \mathrm{rpm}$, Saturación de O2: 92 \% AA (aire ambiente). La paciente estaba en condiciones generales estables, afebril, hidratada, eupneica. En la evaluación de otorrinolaringología (ORL), se evidenció que las mucosas estaban eritematosas y había congestión en ambas fosas nasales, a predominio izquierdo; la orofaringe no presentaba alteraciones. A la exploración cardiopulmonar, se encontró el tórax simétrico, normoexpansible, con ruidos respiratorios presentes en ambos hemitórax, se auscultaron bulosos en los tercios medio y posterior derechos y crepitantes dispersos. Los ruidos cardiacos eran rítmicos y regulares, sin soplos. El abdomen 
característico de una gestante, altura uterina (AU): 32 $\mathrm{cm}$, frecuencia cardiaca fetal (FCF) en $150 \mathrm{lpm}$. No presentaba dinámica uterina. Feto único, longitudinal, dorso izquierdo, cefálico. Al examen ginecológico, los genitales externos estaban normoconfigurados; la evaluación por espéculo mostró la vagina de trayecto libre y el cuello macroscópicamente normal, sin secreciones a través del orificio cervical externo, al tacto, la vagina estaba normotérmica, normotónica, el cuello uterino era largo, posterior, blando y cerrado. No había edema en los miembros inferiores. La exploración neurológica mostró una paciente consciente, orientada en tiempo, espacio y persona, con reflejos osteotendinosos II/IV, sin signos de focalización o déficit.

Se ingresó con los siguientes diagnósticos: 1) Embarazo simple de 33 semanas y 1 día. 2) Neumonía viral por COVID-19 (por clínica), complicada con insuficiencia respiratoria aguda tipo I.
Los resultados iniciales de laboratorio materno se muestran en la tabla 1.

Se practicó tomografía axial computarizada (TAC) de tórax, que reveló múltiples áreas de vidrio esmerilado en parénquima pulmonar bilateral, generalizado, con reforzamiento perihiliar, parahiliar y periférico en forma difusa, con tendencia a la consolidación en hemicampo izquierdo: hallazgos sugestivos de neumonía por COVID-19.

El ecocardiograma transtorácico mostró el pericardio hiperrefrigente engrosado, con derrame pericárdico leve global: hallazgos compatibles con afectación pericárdica por COVID-19.

Al ultrasonido obstétrico del día 29-01-2021 se evidenció un feto único, en cefálica longitudinal, con las siguientes medidas: diámetro biparietal: $84,14 \mathrm{~mm}$, diámetro occipitofrontal: 109,71 mm, circunferencia

Tabla 1. Resultados de exámenes de laboratorio de la madre

\begin{tabular}{|c|c|c|}
\hline Prueba de laboratorio & $29 / 01 / 2021$ & $05 / 02 / 2021$ \\
\hline Hemoglobina (g / dl) & $12,0 \mathrm{~g} / \mathrm{dl}$ & $10,0 \mathrm{~g} / \mathrm{dl}$ \\
\hline Recuento de glóbulos blancos (por $\mathrm{mm}^{3}$ ) & 5,87 (Neut $76 \%$ ) & 6,59 (Neut $70 \%$ ) \\
\hline Recuento de plaquetas (por $\mathrm{mm}^{3}$ ) & 259000 & 259000 \\
\hline Recuento absoluto de linfocitos (por $\mathrm{mm}^{3}$ ) & $15 \%$ & $23 \%$ \\
\hline Dímero D $(\mu \mathrm{g} / \mathrm{mL})$ & $2,80 \mathrm{ug} / \mathrm{ml}$ & $2,10 \mathrm{ug} / \mathrm{ml}$ \\
\hline Glucosa (mg / dL) & $90 \mathrm{mg} / \mathrm{dl}$ & $71 \mathrm{mg} / \mathrm{dl}$ \\
\hline Creatinina (mg / dL) & $0,50 \mathrm{mg} / \mathrm{dl}$ & $0,70 \mathrm{mg} / \mathrm{dl}$ \\
\hline Aspartato aminotransferasa (U / L) & $31 \mathrm{U} / \mathrm{L}$ & \\
\hline Alanina aminotransferasa $(\mathrm{U} / \mathrm{L})$ & $37 \mathrm{U} / \mathrm{L}$ & \\
\hline Sodio $(\mathrm{mEq} / \mathrm{L})$ & $137 \mathrm{mmol} / \mathrm{L}$ & $133 \mathrm{mmol} / \mathrm{L}$ \\
\hline Potasio (mEq / L) & $3,40 \mathrm{mmol} / \mathrm{L}$ & $3,70 \mathrm{mmol} / \mathrm{L}$ \\
\hline Cloruro (mEq / L) & $104 \mathrm{mmol} / \mathrm{L}$ & $105 \mathrm{mmol} / \mathrm{L}$ \\
\hline
\end{tabular}


cefálica: $306,1 \mathrm{~mm}$, circunferencia abdominal: 281,95 $\mathrm{mm}$, longitud femoral: $64,90 \mathrm{~mm}$. El peso fetal, estimado por fórmula de Hadlock y cols. (26) fue de 2084 gramos, en percentil 47 de crecimiento fetal. Se evidenció ecoanatomía fetal conservada, sin la presencia de marcadores ecográficos de síndrome de respuesta inflamatoria fetal.

Al doppler: la arteria uterina derecha con un índice de pulsatilidad: 0,63, en percentil 5-50. La arteria uterina izquierda con un índice de pulsatilidad de 0,68 , en percentil 5-50. La arteria umbilical con un índice de pulsatilidad de 0,85 , en percentil 5-50. La arteria cerebral media con un índice de pulsatilidad de 2,02, en percentil 50-95, con istmo aórtico anterógrado y ductus venoso anterógrado trifásico, con índice de pulsatilidad de 0,6 , en percentil 50. El ultrasonido doppler se concluye como: perfil hemodinámico materno fetal normal. Perfil biofísico fetal de Manning (27): tono fetal: 2 puntos; movimientos respiratorios: 2 puntos; movimientos corporales: 2 puntos; líquido amniótico: 2 puntos. Total: 8/8 puntos.

Se indicó tratamiento médico: Oxigenoterapia, con mascarilla por reservorio a 10 litros por minuto. Remdesivir, vía endovenosa, por 5 días, $200 \mathrm{mg}$ el día 1, luego $100 \mathrm{mg}$ diarios, los días 2 a 5. Dexametasona, $6 \mathrm{mg}$ vía endovenosa por 8 días. Clorhidrato de isoxsuprina, vía oral, $10 \mathrm{mg}$ cada 8 horas. Enoxaparina, 60 mg vía subcutánea diaria. Salbutamol y Budesonida en inhalador, 2 puff cada 8 horas. Loratadina, $10 \mathrm{mg}$ vía oral cada 12 horas.

La paciente permaneció en condiciones generales estables, afebril, hidratada, eupneica. TA: 103/67 mmhg, FC: 98 lpm FR: 22 rpm Sat O2: 96 \%. La evolución fue satisfactoria, tanto materna como fetal, tras 8 días de hospitalización. Se realizó monitoreo fetal anteparto diariamente con resultado reactivo. Se decidió el alta médica, con monitoreo materno fetal más doppler semanal.
El control prenatal sucesivo posterior no reveló alteraciones, doppler dentro de límites normales. Desencadenó trabajo de parto espontáneo, a las 40 semanas de gestación, el día 19/03/2021, con monitoreo fetal intraparto sin alteraciones y frecuencia cardiaca fetal basal de 143 latidos por minuto. Después de 5 horas de conducción de trabajo de parto, se evidenció distocia de dilatación, por lo cual se procedió a realizar cesárea segmentaria, sin complicaciones. Se obtuvo un recién nacido masculino, que lloró y respiró espontáneamente al nacer, peso: 3445 gramos y talla: $50 \mathrm{~cm}$. Se tomó muestra de sangre de cordón umbilical para realizar prueba serológica. Se obtuvo como resultado Inmunoglobulina $(\mathrm{Ig}) \mathrm{G}(+)$ positivo e Ig M (-) negativa. De igual forma se realizó PCR-RT para SARS-CoV-2 que reportó negativo. Se pasó al área de neonatología para evaluación: al examen físico estaba en buenas condiciones generales, piel sonrosada, la evaluación ORL mostró labios indemnes, cuello móvil, sin lesiones, pulmones con murmullo vesicular presente en ambos campos pulmonares, abdomen estaba blando, deprimible, sin visceromegalias, al examen neurológico se encontró tono, llanto y reflejos dentro de límites normales. El diagnóstico proporcionado por el neonatólogo fue: recién nacido a término adecuado para la edad gestacional. (RNAT/ AEG).

\section{DISCUSIÓN}

La diseminación global de la COVID-19 es el problema de salud pública por enfermedad transmisible de mayor riesgo a la gestación, actualmente, en América Latina $(7,8)$.

El espectro de la enfermedad varía desde una infección asintomática hasta un síndrome respiratorio agudo severo. La tasa de mortalidad es de 0 a $6 \%$, dependiendo de diversos factores, principalmente poblacionales (28-30).

La mujer embarazada $y$, por lo tanto, el feto, se consideran población vulnerable, debido a la historia 
natural de la enfermedad, la posible trasmisión vertical y las alteraciones inmunológicas y respiratorias. La COVID-19 ha modificado la vigilancia materna y fetal y se ha vuelto un desafío para el personal de salud implicado en la atención obstétrica $(7,8,20)$.

En los primeros informes de ensayos con reacción en cadena de polimerasa en tiempo real (RT-PCR) para síndrome respiratorio agudo severo (SARS-CoV-2), se evidenció que la trasmisión vertical intrauterina era poco factible (28). Hoy son posibles pruebas serológicas de cordón umbilical y sangre neonatal. Sin embargo, hasta ahora, no está claro si el SARS-CoV-2 puede trasmitirse de la madre al feto, porque su mecanismo principal de contagio es a través de gotitas que se producen cuando una persona infectada tose o estornuda (29). La viremia se encuentra en $1 \%$ de los pacientes con síntomas de COVID-19 y, generalmente, es baja y transitoria, lo que sugiere que es poco probable la transmisión intrauterina del virus (30). Se ha planteado la hipótesis de trasmisión perinatal, pero no está claro si ocurre por vía transplacentaria, intraparto o a través de la exposición del medio ambiente $(31,32)$.

El feto adquiere tempranamente la capacidad de producir inmunoglobulinas séricas. Debido al paso de la IgG materna a través de la placenta, el feto puede mostrar anticuerpos IgG maternos. Sin embargo, los anticuerpos maternos IgM no atraviesan la placenta y su existencia en la sangre fetal es indicativa de una respuesta inmunitaria fetal $(31,32)$.

El caso presentado a modo de punta de iceberg, demuestra la extrema gravedad que puede alcanzar la infección de SARS-CoV-2 durante la segunda mitad del embarazo. Este caso cumple con los criterios propuestos para catalogarlo como de transmisión intrauterina de inmunoglobulinas, considerando los exámenes de anticuerpos en cordón umbilical al nacer, reconfirmados en el primer día de vida. Solo un caso de transmisión transplacentaria de infección por SARS-CoV-2 ha sido reportado previamente por
Vivanti y cols. (31) quienes demostraron la transmisión transplacentaria del virus en un caso grave de infección cercano al parto y que cumplía con todos los criterios propuestos recientemente para infección congénita reciente en el recién nacido vivo: detección del virus por PCR-RT en líquido amniótico y sangre umbilical o neonatal en las primeras 24 horas (32).

En este artículo se reportan 4 casos de afectación moderada-grave materna, diagnosticados por prueba de PCR-RT en el tercer trimestre. De ellas, tres ameritaron hospitalización con tratamiento médico $\mathrm{y}$ evolucionaron de forma satisfactoria, con control prenatal con doppler maternofetal semanal. El cuarto caso descrito, con diagnóstico materno con infección grave, se realizó con un embarazo de 32 semanas por fecha de última regla, que evolucionó de forma satisfactoria, con controles maternos fetales más estudio doppler semanal, con resolución obstétrica por cesárea segmentaria a las 40 semanas de gestación, donde no se demostró compromiso inflamatorio placentario crónico al momento de la resolución y con la evidencia de paso trasplacentario de inmunoglobulinas de tipo $\mathrm{G}$ (positiva). Las imnunoglobulinas tipo $\mathrm{M}$, que le son únicas al feto y no traspasan la barrera placentaria, estaban negativas.

Se requieren más estudios que aborden el tema de la transmisión intrauterina alejada del parto, así como sus consecuencias sobre la salud materna, fetal y neonatal.

\section{CONCLUSIÓN}

La transmisión materno fetal del virus SARS-CoV-2 no se detectó en el caso reportado, aunque el recién nacido tuvo una serología con IgG positiva y IgM negativa al momento de nacer y a las 24 horas. Se comprueba así, la capacidad que tiene la madre de transmitirle anticuerpos al recién nacido a horas de su 
nacimiento y que le brinda la protección para prevenir la infección neonatal.

\section{REFERENCIAS}

1. Velavan TP, Meyer CG. The COVID-19 epidemic. Trop Med Int Health. 2020; 25(3):278-280. doi: 10.1111/ tmi. 13383 .

2. Organización Mundial de la Salud [Internet]. Ginebra: Coronavirus; 2020 [consultado el 2 de abril de 2021]. Disponible en: https://www.who.int/es/health-topics/ coronavirus.

3. International Committee on Taxonomy of Viruses [Internet]. 9th Report Coronaviridae; 2011 [consultado 6 de febrero de 2021]. Disponible en: https://talk. ictvonline. org/ictv/reports/ictv_9th_report/positive/ sense/rna/viruses/2011/w/posrna_viruses/222/ coronaviridae.

4. Song $\mathrm{Z}, \mathrm{Xu} \mathrm{Y}$, Bao L, Zhang L, Yu P, Qu Y, et al. From SARS to MERS, Thrusting Coronaviruses into the Spotlight. Viruses. 2019; 11(1):59. doi: 10.3390/ v11010059.

5. Huang C, Wang Y, Li X, Ren L, Zhao J, Hu Y, et al. Clinical features of patients infected with 2019 novel coronavirus in Wuhan, China. Lancet. 2020; 395(10223): 497-506. doi: 10.1016/S01406736(20)30183-5.

6. Dashraath P, Wong JLJ, Lim MXK, Lim LM, Li S, Biswas A, et al. Coronavirus disease 2019 (COVID-19) pandemic and pregnancy. Am J Obstet Gynecol. 2020; 222(6):521-531. doi: 10.1016/j.ajog.2020.03.021.

7. Carvajal A, Márquez D. Nuevo coronavirus (SARSCoV-2) y embarazo. Rev Obst Ginecol Venez [Internet]. 2020 [consultado 2 de abril de 2021]; 80(1):53-63. Disponible en: http://www.sogvzla. org.ve/sogvzla $20186 / \mathrm{cms} / \mathrm{svcobtenerpdfrevista.}$ php?id $=0000000090 \&$ tipo $=$ normal $\&$ fila $=9$

8. Gómez J, Cabrera C, Guillén A, Cuadra C, Correa L; "Red COVID-19 y Gestación". Red Covid-19 y gestación. Rev Obstet Ginecol Venez [Internet] 2020 [consultado 2 de abril de 2021]; 80 (Sup1): S56-S69. Disponible en: http://www.sogvzla. org.ve/sogvzla $20186 / \mathrm{cms} / \mathrm{svcobtenerpdfrevista.}$ php?id $=0000000098 \&$ tipo $=$ normal $\&$ fila $=5$

9. Schwartz DA, Graham AL. Potential Maternal and Infant Outcomes from (Wuhan) Coronavirus 2019nCoV Infecting Pregnant Women: Lessons from SARS, MERS, and Other Human Coronavirus Infections. Viruses. 2020; 12(2):194. doi: 10.3390/v12020194.
10. Chen H, Guo J, Wang C, Luo F, Yu X, Zhang W, et al. Clinical characteristics and intrauterine vertical transmission potential of COVID-19 infection in nine pregnant women: a retrospective review of medical records. Lancet. 2020; 395(10226):809-815. doi: 10.1016/S0140-6736(20)30360-3.

11. Herrera M, Arenas J, Rebolledo M, Baron J, De Leon J, Yomayusa N, et al. Embarazo e infección por coronavirus COVID-19. Fundación Internacional de Medicina Materno Fetal; 2020 [actualizado 1 de abril de 2020; consultado 2 de abril de 2021]. Disponible en: https://www.flasog.org/static/COVID-19/FIMMF.pdf

12. López M, Goncé A, Meler E, Hernández S, Cobo T, Guirado L, et al. Protocolo: Coronavirus (COVID-19) y Gestación [Internet]. Barcelona: Clinic Barcelona Hospital Universitar, Centro de Medicina Fetal Neonatal de Barcelona; 2020 [actualizado 8 de marzo de 2021; consultado 1 de mayo de 2021]. Disponible en: https://medicinafetalbarcelona.org/protocolos/es/ patologia-materna-obstetrica/covid19-embarazo.pdf

13. Hernández P, Canache L. Covid-19 y la programación fetal. Rev Obst Ginecol Venez [Internet]. 2020 [consultado 1 de mayo de 2021]; 80 (Supl 1): S70-S78. Disponible en: http://www.sogvzla. org.ve/sogvzla2 $0186 / \mathrm{cms} / \mathrm{svcobtenerpdfrevista.}$ php? $\mathrm{id}=0000000098 \&$ tipo $=$ normal $\&$ fila $=9$

14. Fuenzalida J, Theodor M. Solari C, Poblete J, Carvajal J, Vera C, et al. Guía de Manejo. COVID-19 Embarazo [Internet]. Santiago de Chile: Facultad de Medicina Pontificia Universidad Católica de Chile; 2020 [actualizado 14 de abril de 2020; consultado 1 de mayo de 2021]. Disponible en: https://medicina. uc.cl/wp-content/uploads/2020/04/Gui\%CC\%81ade-Manejo-COVID-19-y-Embarazo-SegundaActualizacio\%CC\%81n.pdf

15. Jeong SY, Sung SI, Sung JH, Ahn SY, Kang ES, Chang YS, et al. MERS-CoV infection in a pregnant woman in Korea. J Korean Med Sci. 2017; 32(10):1717-1720. doi: $10.3346 / \mathrm{jkms} .2017 .32 .10 .1717$.

16. Ng WF, Wong SF, Lam A, Mak YF, Yao H, Lee $\mathrm{KC}$, et al. The placentas of patients with severe acute respiratory syndrome: a pathophysiological evaluation. Pathology. 2006 Jun;38(3):210-8. doi: 10.1080/00313020600696280.

17. Special Expert Group for Control of the Epidemic of Novel Coronavirus Pneumonia of the Chinese Preventive Medicine Association. [An update on the epidemiological characteristics of novel coronavirus pneumonia (COVID-19)]. Zhonghua Liu Xing Bing Xue Za Zhi. 2020; 41(2):139-144. Chinese. doi: 
10.3760/cma.j.issn.0254-6450.2020.02.002.

18. Goodnight WH, Soper DE. Pneumonia in pregnancy. Crit Care Med. 2005; 33(10 Suppl):S390-7. doi: 10.1097/01.ccm.0000182483.24836.66.

19. Pérez J, Márquez D, Lugo C, Veroes J, Cortés R, Di Muro J, et al. Embarazada y COVID-19. Guía provisional de la Sociedad de Obstetricia y Ginecología de Venezuela. Rev Obst Ginecol Venez [Internet]. 2020 [consultado 1 de mayo de 2021]; 80(Supl 1): S3-S29. Disponible en: http://www.sogvzla. org.ve/sogvzla20186/cms/svcobtenerpdfrevista. php?id $=0000000098 \&$ tipo $=$ normal $\&$ fila $=3$

20. Sociedad Venezolana de Ultrasonido en Medicina [Internet]. Caracas: Consenso AVUM COVID-19 en gestantes; 2020 [consultado 2 de abril de 2021]. Disponible en: http://avum.org/consenso-avum-covid19-en-gestantes/

21. Ministerio del Poder Popular para la Salud, Comité Terapéutico COVID-19 [Internet]. Caracas: Guía para el manejo y tratamiento de contactos y pacientes con COVID-19; 2021 [actualizado 26 de abril de 2021; consultado 01 de mayo de 2021]. Disponible en: https://drive.google.com/file/d/1XLTUt4W1aIjLf34y AZWrCOR9O6dA4w4c/view? usp=sharing.

22. Sanford Guide Web Edition [Internet]. Sperryville, VA: COVID-19, SARS CoV-2; 2021 [actualizado 20 de abril de 2021; consultado 01 de mayo de 2021]. Disponible en: https://webedition.sanfordguide.com/ en/sanford-guide-online/disease-clinical-condition/ coronavirus

23. Organización Mundial de la Salud [Internet]. Ginebra: Manejo clínico de la COVID-19. Orientaciones evolutivas; 2021 [actualizado 25 de enero de 2021; consultado 22 de mayo de 2021]. Disponible en: https:// apps.who.int/iris/bitstream/handle/10665/340629/ WHO-2019-nCoV-clinical-2021.1-spa.pdf.

24. Organización Mundial de la Salud [Internet]. Ginebra: Pruebas diagnósticas para el SARS-CoV-2. Orientaciones provisionales; 2020 [actualizado 11 de septiembre de 2020; consultado 22 de mayo de 2021]. Disponible en: https://apps.who.int/iris/bitstream/ handle/10665/335830/WHO-2019-nCoV-laboratory2020.6-spa.pdf

Vol. 81, $\mathrm{N}^{\mathrm{o}} 2$, junio 2021
25. Carvajal A, Cabrera C, Guillén A, Cuadra C, Correa L. Red COVID-19 y Gestación. Rev Obstet Ginecol Venez [Internet]. 2020 [consultado 22 de mayo de 2021]; 80 (Sup1): S36 - S44. Disponible en: http://www.sogvzla. org.ve/sogvzla20186/cms/svcobtenerpdfrevista. php? $i d=0000000098 \&$ tipo $=$ normal $\&$ fila $=5$

26. Hadlock FP, Harrist RB, Carpenter RJ, Deter RL, Park SK. Sonographic estimation of fetal weight. The value of femur length in addition to head and abdomen measurements. Radiology. 1984; 150(2):535-540. doi: 10.1148/radiology.150.2.6691115.

27. Manning FA. The use of sonography in the evaluation of the high-risk pregnancy. Radiol Clin North Am [Internet]. 1990 [consultado 22 de mayo de 2021]; 28(1):205-216. Disponible en: https://pubmed.ncbi. nlm.nih.gov/2404302/

28. Rasmussen SA, Smulian JC, Lednicky JA, Wen TS, Jamieson DJ. Coronavirus Disease 2019 (COVID-19) and pregnancy: what obstetricians need to know. Am J Obstet Gynecol. 2020; 222(5):415-426. doi: 10.1016/j. ajog.2020.02.017.

29. Zeng H, Xu C, Fan J, Tang Y, Deng Q, Zhang W, et al. Antibodies in Infants Born to Mothers With COVID-19 Pneumonia. JAMA. 2020; 323(18):1848-1849. doi: 10.1001/jama.2020.4861.

30. Shah PS, Diambomba Y, Acharya G, Morris SK, Bitnun A. Classification system and case definition for SARS-CoV-2 infection in pregnant women, fetuses, and neonates. Acta Obstet Gynecol Scand. 2020; 99(5):565-568. doi: 10.1111/aogs.13870.

31. Vivanti AJ, Vauloup-Fellous C, Prevot S, Zupan V, Suffee C, Do Cao J, et al. Transplacental transmission of SARS-CoV-2 infection. Nat Commun. $2020 \mathrm{Jul}$ 14;11(1):3572. doi: 10.1038/s41467-020-17436-6.

32. Alzamora MC, Paredes T, Caceres D, Webb CM, Valdez LM, La Rosa M. Severe COVID-19 during Pregnancy and Possible Vertical Transmission. Am J Perinatol. 2020; 37(8):861-865. doi: 10.1055/s-0040-1710050.

Recibido 4 de abril de 2021 Aprobado 15 de mayo de 2021 